\title{
III. Ueber gleichmässigen (pendelartigen) Rhythmus der Herztöne und dessen Beziehung zum Galopprhythmus.')
}

Von Dr. med. J. Pawinski,

Primararzt am Kindlein Jesu Hospital zu Warschau.

Schon lange erregte meine Aufmerksamkeit eine besondere Art von Rhythmus der Herztöne, den ich, weil derselbe an den Rhythmus der Pendelbewegungen vollständig erinnert, als pendelartigen zu bezeichnen geneigt wäre. Diese obgleich keineswegs exacte Bezeichnung erklărt doch am besten die Sachverbältnisse. Die Physiker nennen einen ähnlichen Rhythmus einen gleichartigen.

Dieser Rhythmns zeichnet sich hauptsächlich dadurch aus, dass die Zeit, die zwischen dem ersten und dem zweiten Tone verläuft, ungefähr derjenigen entspricht, welche denzweiten Ton von dem nächsten ersten trennt-mit anderen Worten die kleine Pause kommt der grossen gleich. Unter physiologischen Verhältnissen sind die Intervalle zwischen den Herztönen bei einem Erwachsenen einander nicht gleich - es giebt eine grosse und eine kleine Pause. Fin solcher Rhythmus ändert seinen Charakter weder bei beschleunigter, noch bei verlangsamter Herzaction - was hauptsächlich darauf zu beziehen ist, dass die Systole weniger Zeit beansprucht als die Diastole.

Als zweites Merkmal des pendelartigen Rhythmus ist die Verstärkung der Herztöne und die Gleichartigkeit derselben in akustischer Beziehung zu betrachten.

Unter normalen Verhältnissen ist der erste Ton fül gewöhnlich etwas länger, der zweite kürzer, wie abgeschnitten. Jedoch bieten dieselben je nach der Auscultationsstelle manche Verschiedenheiten bezüglich der Accentuation: über der Herzspitze fällt der Accent auf den ersten Ton (daher Aehulichkeit mit Trochaeus), über der Herzbasis ist es umgekehrt, der zweite Ton accentuirt (Jambus). Auch in dieser Beziehung können manche Abweichungen stattfinden: entweder kommt die Accentuation keinem Tone zu, oder dieselbe fällt gleichzeitig auf den ersten und zweiten Ton. Der letztere Fall findet nämlich bei dem pendelartigen Rhythmus an der Herzspitze statt. Es liegt nahe, aus deın eben Geschilderten den Schluss zu ziehen, dass der pendelartige Rhythmus nichts weiteres darstellt, als die jüngst von Huchard beschriebene und von Stokes bereits früher erwähnte Embryocardie - denn in der That haben wir es in beiden Fällen mit einem foetalen Rhythmus zu thun. Indessen unterscheidet sich der pendelartige Rhythmus von dem dem Foetus zukommenden dadurch, dass die Stärke der Herztöne bedeutend grösser ist, ausserdem noch dadurch, dass derselbe nicht nur bei frequenten, sondern auch bei gewöhnlichem, ja sogar bei verlangsamtem Pulse erscheinen kann. Später werden wir sehen, unter welch' verschiedenen Bedingungen der erwähnte Rhythmus zustande kommt, und von welch' verschiedener klinischer Bedeutung derselbe sein kann.

1) Vortrag für den X. internationalen medicinischen Congress zu Berlin 1890 bestimmt. 
Was die Krankheiten, in denen mir dieser Rhythmus begegnetwar, anbetrifft, so stehen die Erkrankungen der Nieren obenan, dann kommt die Arteriosclerose und die Herzhy pertrophie.

I. Nierenkrankheiten. Vor allem kommen hier die verschiedenen Formen der chronischen Eutzündungen in Betracht -bei den acuten Formen ist dies sehr selten der Fall. Der pendelartige Rhythmus tritt am häufigsten in den gemischten Formen (Nephritis mixta) oder in der weniger vorgeschritteuen interstitiellen Form (N. interstitialis) auf - dort aber, wo der bindegewebige Process in deu Niereu weit vorgerïckt, die Herzhypertrophie bedeuteud und die Herzaction sehr energisch ist, dort habe ich den in Rede stehenden Rhythnuus vermisst.

Die Herzdimensionen waren in solchen Fällen entweder normal, oder die linke Kammer hat eine gewisse Vergrösserung erlitten, oder schliesslich die Herzhypertrophie ist bedeutend, aber der Muskel fällt der Erschöpfung anheim (Hyposystolia).

Zuweilen tritt dieser Rhythmus im Verlauf von Nierenkranklieiten auf, nachdem in Folge starker körperlicher Emotion eine Ueberanstrengung des Herzens eingetreten ist. Dann kommt derselbe zum Vorschein entweder in der ersten Phase der Ueberanstrengung oder erst später, wenn nach vorausgegangener Dilatation das Herz seine frühere Leistıngsfähigkeit wiedergewinnt. Dies hängt ınit der grösseren oder geringeren Leistungsfähigkeit des Herzmuskels zllsammen.

Schliesslich erscheint der pendelartige Rhythmus in Fällen von chronischer Nephritis mit hochgradig verninderter Diurese und verlangsamtem Herzrhythmus, wenn die Harnmenge spontan oder kiinstlich durch Darreichung von Strophanthus, Coffein u. s. w. zuzunehmen beginnt und der Rhythmus fiequeut wird.

Da der pendelartige Rhythmus häufig bei den latent verlaufenden Krankheitsprocessen in den Nieren eintritt, so gewinnt derselbe für den Klinicisten eimen hohen diagnostischen Werth.

II. Die in Bezug anf die Häufigkeit des Vorkommens des pendelartigen Rhythmuıs nächststehende Grıppe von Krankheiten ist die Arteriosclerose und die Herzhypertrophie. Dieselbe ist keineswegs eine constante Erscheinung und hält zuweilen nur sehr knrze Zeit an. Physische Emotionen, sowie Gemuithsbewegungen sind als prädisponirende Nomente anzısehen. Den höheren Graden von Hypertrophie der linken Kamner kommt für gewöhnlich der gleichmässige Rhythmus nicht zu

Hierher sollen auch diejenigen Fälle gerechmet werden, in denen der gleichartige Rhythmus bei Kranken mit gesteigerter Gefässspannung ohne Arteriosclerose zustande kommt. Dies betraf sehr oft jugendliche Individuen mit enormer Erregbarkeit des vasomotorischen Systems (Angioneurosis). Die gesteigerte Gefässspannung wird in solchen Fällen anf die sehr oft reflectorisch zustande kommende Contraction del kleinsten Gefässe be'ogen.

Bei Morbus Basedowii gehört der pendelartjge Rhythmus zil den seltenen Erscheinungen.

III. Mehrfach vermochte ich den in Rede stehenden Rhythmus bei tuberculösen Individuen zu constatiren, nänllich dann, wenn die tuberculöse Infiltration die rordere Partie der linken Lunge ergriff, nnd das Her\% in seiner oberen und linken Grenze von einer indurirten Masse unngeben war. Solche Individnen fieberten fiil ${ }^{\circ}$ ewöhnlich nicht. Der Puls pflegt in solchen Fällen ziemlich gespannt, fïr gewöhnlich beschleunigt znl sein, die Herztöne sind ziemlich stark und besitzen infolge der angrenzenden indurirten Massen, die einen gnten Leiter darstellen, einen metallischen Beiklang.

Als Ursache des pendelartigen Rhythmus mögen diejenigen Hindernisse geltell, welche dem Herzen seitens der wenig nachgiebigen luftleeren Massen entgegenstehen, sowie eine gewisse $\mathrm{Er}$ regbarkeit des vasomotorischen Systems.

Man kann im allgemeinen behaupten, dass dieser Rhythmus von folgenden Symptomen seitens des Circulationssystems begleitet wird:

1. voll einer gesteigerten Spannung im Aortensystem,

2. von einer gesteigerten Herzaction und

3. von eiuem Pulsschlag, der häufiger etwas beschlennigt als verlangsamt ist.

Es liegt jetzt die Frage uahe, wodurch die Entstehnng des pendelartigen Rhythmus zu erklären ist.

Es wurde bereits erwähut, dass die Systole bei einem Erwachsenen viel weniger Zeit be $2 n s p r u c h t$, als die Diastole (Heyden berechnete bei einem Pulsschlage von 90 in einer Minute, die Zeit der Systole auf 0,250 Secunden, der Diastole auf 0,666 Secunden. Donders giebt bei 74,4 Pulsfrequenz - die ganze Herzrevolution $=$ 0,801 Secunden - fiil die Zeit der Systole 0,327 Secunden, fïr die der Diastole 0,474 Secunden an. Lando is berechnete bei 55 Pulsschlägen in einer Minute -- 1,333 Secunden der ganzen Herzrevolution - die Zeit der Systole auf $0,309-0,346$ Secunden, die del Diastole 822-0,782 Secunden.)
Es ist auch wohl bekannt, dass die Zeit der Herzsystole in Vergleich mit derjenigen del Diastole nur sehr unbedentenden Schwankungen unterliegt. Komint die kleine Pause bei dem pendelartigen Rhythmus der grossen gleich, so kann dies entweder von der Verkiirzung der Zeit der Diastole bei nnverändert gebliebener Zeit der Systole herriihren, oder die Zeit der Systole wird bei nnverändert bleibender Diastole verlängert, oder beide Zeiten nnterliegen gewissen Schwankungell, wobei jedoch die Verlängerung der Systolezeit die hervorragende Rolle spielt.

Die erste und zweite Voranssetzung musste auf Grund cardiographischer Bestimmungen zurïickgewiesen werden. Dieselben haben nämlich bewiesell, dass, obgleich die Zeit der Herzdiastole (Ventrikeldiastole + Pause + Vorhofscontraction) etwas kür\%er wurde, so war dieselbe doch nicht von so hohem Grade, dass man dadurch allein die Gleichheit der Pause zu erklären im Stande wäre. Dagegen erlitt die Zeit der Herzsystole eine hochgradige Verlängernug.

Die Zeit der Herzsystole besteht ans zwei Abschnitten:

1. Als der Zeit zwischen dem Beginn der Systole und dem Maximun ilurer Intensität, ans der sogenannten Verschlusszeit (Martius), d. h. ans der Zeit von dem Momente des Verschlusses der Mitralklappen bis zur Eröffnung der Semilunarklappen und

2. Aus der Zeit zwischen der Eröffnung der Semilunarklappen bis zur Schliessung derselben, aus der Austreibungszeit (Martius).

Jeder dieser Zeitabschnitte vermag natïrlich zur Verlängerung der Systole beizutragen. Allein ich kam auf Grund cardiographischer Bestimmungen zu der Ueberzengung, dass zur Verlängerung der Zeit der Systole hauptsächlich die Vergrösserıng der ersten Zeitabschuitte beigetragen hat. So fielen bei an Arteriosclerose mit mässiger Hypertrophie der linken Kammer leidendem Individunm bei einem Pulsschlag von 76 in eiuer Minute, also bei einer Herzrevolution $=0,789$ Secunden, auf den ersten Zeitabschnitt (Verschlusszeit) 0,190 Secunden, anf deu zweiten 0,156 Secunden. Es dauerte also die Herzsystole oder die kleiue Panse 0,346 Secunden, die Diastole oder die grosse Pause 0,443 Secunden, Differenz $=0,097$ Secunden. In einem Falle von Mischform von Sephritis nahm bei einem Pulsschlage von 94 in einer Minute (Herzrevohition $=0,636$ Secunden) die Systole 0,306 Secunden $(0,176+0,130$ Secunden $)$, die Diastole 0,332 Secunden in Anspruch, die Differenz betring also $0,026 \mathrm{Se}-$ cunden. Nur ausnahmsweise kanu es vorkommen, dass die Zeit der Systole genan derjenigen der Diastole entspricht, dass also die Pausen vollständig mit einander iibereinstinmen, jedoch ist diese mathematische Genauigkeit, da unser Gehörsinn diese kleinen Zeitschwankungen, zumal bei gleicher Stärke beider Töne, walırzı. nehmen nicht vermag, keineswegs fiir die Entstehung des pendelartigen Rhythmus nothwendig. Es sei hier erwähnt, dass der zweite Zeitabschnitt der Systole, d. h. die Zeit des Einströmens des Bhıtes in die Aorta, ebenfalls eine Verlängerung erfahren hat, allein diese fand in viel geringerem Grade als im ersten Zeitabschnitte statt. Unter normalen Verhältuissen beansprucht der zweite Abschnitt der Systole stets mehr Zeit, als der erste; umgekehrt verhält es sich in Fällen, in denen ein gleichmässiger Rhythmus wahrgenommen wird.

Die Verlängerung der Zeit der Systole wird uns sofort klar, wenu wir im Auge behaltell, dass eben in den oben genanntell Krankheitsformen, hauptsächlich bei Nierenleiden, eine betrïchtliche Drucksteigeruıg iu der Aorta stattfindet, infolge deren die linke Kammer, uı die im Aufangsstïcke dei Aorta vorhandenen Hindernisse zu ïberwinden, d. h. $11 \mathrm{~m}$ die Semilunarklappen zn eröffnell, mehr Zeit beansprucht, als nnter gewöhnlichen Druckverhältnissell. Unsere aus der Zeitdaner der Systole und der Diastole unter patho. logischeu Verhältnissen gezogeneu Schln̈sse findeu ihıe vollständige Bestätigung iu der Arbeit vou Martius. ${ }^{1}$ ) Dieser Autor constatirte nämlich, indem er die Bestandtheile der Cardiogramme bei gesunden Individuen analysirte, dass die Zeitdauer der beiden Abschnitte der Herzsystole (Verschluss- und Austreibungszeit) manchen Schwankuugen anheimfällt, was ja seiner Meinung nach auf die in Aortasystem herrschenden Druckverhältnisse zurückzuführen sei. Derselbe Autor konnte sich zul Fieberzeit (in der der Aortendruck für gewöhnlich sinkt) bei einem 21jährigen an Pneumonic erkrankten Individnum überzengen, dass der erste Abschnitt der Systole, die Verschlusszeit 0,07 Secunden betring. während der zweite Abschnitt, die Austreibungszeit, zweimal so viel Zeit, nämlich $0,11-0,13$ Secunden in Anspruch nahm. Umgekehrt verhielt es sich bei einem alten Manı init Arteriosclerose - der Blutdruck war bei demselben gesteigert, weshalb der erste Abschnitt der Systole viel länger, nämlich 0,15 bis 0,16 Secunden, der zweite 0,08 Secunden dauerte.

In manchen Fällen von Stenose des Aortenostin un erfảhrt die Zeit der Systole eine beträchtliche Verlängerung, so dass dieselbe die der Diastole ïbertrifft - in diesen Fällen wird haupt-

1) Graphische Untersuchungen ïber die Herzbewegung. Zeitschrift für klin. Med. 13 Bd. 3. 4. 5. Heft. Berlin 1887. 
sächlich der zweite Abschnitt der Systole, d. h. die Zeit zwischen der Eröffnung und Schliessung der Semilunarklappen, verlängert. Bei einem Kranken mit Stenosis ostii art. sinistr. mit rhythmischem Pulsschlage und einer Herzbewegung von ca. 0,781 Secunden betrug, nach eigener Beobachtung, die Zeit der Systole 0,471 Secunden, die der Diastole 0,310 Secunden, wobei auf den ersten Abschnitt der Systole 0,213 Secunden, auf den zweiten 0,256 Secunden kam. Dieses Ergebniss wird leicht erklärt, sobald wir in Betracht ziehen, dass der Blutstrom im Anfangsstücke der Aorta auf eine stenosirte Stelle stösst, weshalb derselbe mehr Zeit zum Einströmen in die Aorta beansprucht.

Obgleich die Beschaffenheit des Pulses, nämlich die Stärke und Grösse der Welle gewissermassen von dem ersten Abschnitte der Systole beeinflusst wird, so tritt doch die Abweichung im Charakter des Pulses viel prägnanter hervor bei Veränderungen, welche die Zeit des Ausströmens des Blutes aus der Kammer in die Aorta erfährt (wie das bei Sten. ostii arter. sin.). Aus diesem Grunde entbehrt der Puls bei pendelartigem Rhythmus diejenigen charakteristischen Merkmale, welche er bei Sten. ost. art. sin. zu bieten pflegt (pulsus tardus und gewöhnlich rarus). Infolge des gesteigerten Blutdruckes ist die Rückstosselevation geringer, dagegen treten die Elasticitätselevationen viel deutlicher hervor, was jedoch je nach der Beschaffenheit der Arterienwandungen gèwissen Modificationen anheimfallen kann. Jedoch zeigt der Pulsschlag nicht immer denselben Charakter - hat das Arteriensystem seine Leistungsfähigkeit nicht eingebüsst, wie es im jugendlichen Alter der Fall ist, so habe ich trotz hochgradiger Spannung mehrfach das Bild des leicht dicroten Pulses erhalten. Bei übermässiger Spannung kommt die Natur dem Organismus zur Hülfe - der compensatorische nervöse Apparat tritt in seine Rechte ein, indem er eine Erschlaffung der Gefässe, die der Blutdruck herabsetzt, bewirkt. (Ich habe mich mehrfach davon überzeugen können, indem ich den Einfluss des Strophanthus auf Herz und Pals studirte. Das gleiche beobachtete Oertel beim Bergsteigen.) Dadurch wird die Kraft, die vom Herzen zur Eröffnung der Semilunarklappen verlangt wird, geringer, der gleichmässige Rhythmus verschwindet, indem er dem gewöhnlichen Rhythmus Platz macht.

Es leuchtet also aus dem oben Angegebenen ein, dass, obgleich der pendelartige Rhythmus im wesentlichen gleich der Embryocardie einen gleichartigen (foetalen) Rhythmus darstellt, so ist er doch in klinischer Beziehung ein vollständiger Gegensatz derselben. Bei dieser constatiren wir eine gesteigerte Herzaction, laute Töne, Drucksteigerung im Aortensystem, bei jener ist die Herzaction schwach, fast erlöschend, die Töne schwach, und der Blutdruck bedeutend herabgesetzt. Die .Embryocardie ist ein sehr ungünstiger, sehr oft den heranrückenden Tod verkündigender Vorläufer, dagegen deutet der pendelartige Rhythmus hauptsächlich auf eine Zunahme der Hindernisse in der Circulation und einen reactionsfähigen Herzmuskel hin. Derselbe besitzt nicht nur einen diagnostischen Werth, er ist sogar von hoher therapeutischer Bedeutung. Vor allem muss eine Beseitigung der Circulationshindernisse, eine Herabsetzung der Gefässspannung erfolgen, erst dann kommt die Steigerung der Triebkraft des Herzens in Betracht.

Wir gehen jetzt zur Betrachtung der Beziehung des pendelartigen Rhythmus, zum Galopprhythmus über.

Unter Galopprhythmus verstehen wir einen Rhythmus, der aus drei Tönen besteht, von denen zwei zu den gewöhnlichen gehören und der dritte ein zusätzlicher ist. Die Mehrzahl der Autoren betrachten ihn als eine Verdoppelung des ersten Tones, andere dagegen, namentlich Fraentzel, rechnen ihn zum zweiten Tone hinzu. Was die Accentuation anbelangt, so bält Fraentzel für einen Galopprhythmus nur einen solchen Rhythmus, bei dem der Accent auf den ersten diastolischen Ton fällt.

Was die Grenze des geschilderten Rhythmus anbetrifft, so kam ich auf Grund zahlreicher Beobachtungen zu dem Schlusse, dass als Ausgangspunt des Galopprhythmus gerade der pendelartige Rhythmus anzusehen sei.') Zu diesem Schlusse gelangte ich durch die Berbachtung, dass bei Patienten, bei denen der Rhythmus der Herztone ein gleichartiger war, derselbe zuweilen während der Auscultation, sobald die Herzaction bald unter dem Einfluss psychischer Momente, bald durch tiefere Athmung und rascheres Geben beschleunigt und der Puls frequenter wurde, die Charaktere des Galopprhythmus annahm. Dann erschien anstatt zweier durch gleiche Pausen von einander getrennter

1) Von den Autoren giebt nur einer, nämlich D'Espine, an (Essay de Cardiographie elinique. Revue de Médecine. Paris 1882. Janvier et Fevrier), dass in manchen Fällen von Nephritis eine Verdoppelung des ersten Tones nicht stattfindet, sondern derselbe rauh und verlängert erscheint (le premier bruit renfé). Derselbe soll eigentlich für den Ausgangspunkt des Galopprhythmus gelten.
Töne ein dritter Ton, der unmittelbar dem ersten vorausging, wobei der Accent entweder auf den ersten Ton fiel oder, was häufiger der Fall war, auf dem zweiten sich markirte. Es kam auch vor, dass der dritte zusätzliche Ton $_{\text {on }}$ bei besleunigter Herzaction nicht auftrat, sondern dass der erste Ton den Accent trug. Andererseits ging der Galopprhythmus unter dem Einfluss von Digitalis, Strophanthus, Coffein oder Jodkali unter gleichzeitiger Abnahme der Frequenz der Herzschläge in den pendelartigen, zuweilen auch, obgleich viel seltener, in den gewöhnlichen Rlythmus über. Auf Grund dessen glaube ich, dass der zusätzliche Ton in einem.innigen Zusammenhange mit dem ersten Ton oder, um mich genauer auszudrücken, mit der Systole der linken Kammer sich befindet. Die Entstehung des Galopprhythmus ist wahrscheinlich so zu erklären, dass die Herzsystole sich in zwei Zeitabschnitten tollzieht, was bereits früher Leyden und D'Espine in seiner ausführlichen Arbeit (l. c.) zu beweisen sucht.

Wir haben bereits früher erwähnt, dass der pendelartige Rhythmus sich dadurch auszeichnet, dass die Systole, da von der Kammer, um die in der Aorta herrschende Spannung zu überwinden, mehr Zeit verlangt wird, mehr Zeit in Anspruch nimmt, als es gewöhnlich der Fall zu sein pflegt. Sobald aber die Herzcontractionen an Frequenz zuzunehmen und das Herz lebhafter zu schlagen beginnt, dann füllt sich die linke Kammer sofort mit Blut, es beginnt die Systole, die aber für die Eröffnung der Semilunarklappen nicht ausreicht, das Herz macht grössere Anstrengungen es tritt ein neuer Anprall der Blutmasse gegen die Atroventricularklappen ein; dadurch erscheint der erste Ton wie verdoppelt, und der Accent fällt hauptsächlich auf den zweiten Ton.

Der Galopprhythmus geht entweder in den pendelartigen, was für ein günstiges Zeichen betrachtet wird, oder in eine Arbythmie über, führt zuweilen zur Herzdilatation und vermag schliesslich in eine Herzlähmung überzugehen. Dies hängt augenscheinlich mit der Leistungsfähigkeit des Herzmuskels und seiner nervösen Ganglien zusammen.

Was die klinische Bedeutung des Galopprhythmus anbetrifft, so betrachte ich denselben im Gegensatz zu der Mehrzahl der Autoren nicht für eine absolut ungünstige Erscheinung. Gleich dem gleichmässigen Rhythmus gilt derselbe nicht so für ein Zeichen von Herzschwäche, als vielmehr für ein Symptom gesteigerten Blutdrucks, gesteigerter Hindernisse, welche das Herz zu überwinden hat, also gegenüber denen dasselbe, wenn es noch einen gewissen Kraftvorrath besitzt, den Kampf oft siegreich besteht. Dass der pendelartige Rhythmus eine bessere Prognose als der Galopprhythmus gestattet, ist selbstverständlich. 